\title{
UNA ESTIMACIÓN DE RESPUESTA EN CASO DE SISMO GRAVE
}

\author{
AN ESTIMATE OF RESPONSE IN CASE OF SEVERE EARTHQUAKE
}

UMA ESTIMATIVA DE RESPOSTA EM CASO DE UM GRAVE SISMO

\author{
GUSTAVO RAMÍREZ AMAT', GIAFFAR BARQUET ABI HANNA', CARLOS SANTANA VÉLIZ1
}

${ }^{1}$ Universidad Católica de Santiago de Guayaquil, Guayaquil, Ecuador

Resumen

Objetivos: describir las características generales que tienen los servicios de emergencia de los hospitales de la ciudad de Guayaquil; el flujo de pacientes, las proporciones de internamiento y cirugías de emergencia, las tasas de ocupación y la disponibilidad de camas, estimando la posibilidad de atender un aumento súbito de la demanda por un desastre natural, modelar una situación de incremento estimando la capacidad de atención en función de la disponibilidad de camas. Diseño del estudio: transversal para la descripción de los servicios de emergencia de cinco hospitales de la provincia del Guayas y se simuló un incremento lineal de la demanda de servicios para evaluar la capacidad de atención. Resultados: las atenciones de emergencia se han incrementado significativamente en los últimos cinco años, mientras que las tasas de internamiento por emergencia han disminuido; la disponibilidad de camas por habitante no se ha incrementado y el número de camas disponibles está poco más arriba de 200 por día en promedio. Conclusiones: los hospitales que tendrían mayor porcentaje de capacidad de atención serían el León Becerra, el Naval y el Militar. Los hospitales del sector del sur y oeste de la ciudad, son los que tendrían menor capacidad para atender un mayor volumen de pacientes.

PALABRAS CLAVE: servicios médicos de urgencia, administración hospitalaria, capacidad de camas en hospitales.

Abstract

Objectives: to describe the general characteristics the emergency services of the hospitals in the city of Guayaquil have; patient flow, rates of hospitalization and emergency surgeries, occupancy rates and bed availability, estimating the possibility of facing a sudden increase in demand due to a natural disaster, model a situation of increase estimating service capacity according to bed availability. Study design: cross-sectional study for the description of the emergency services of five hospitals in the province of Guayas and a linear increase in demand for services to assess service capacity was simulated. Results: emergency care demand has increased significantly over the past five years, while rates of emergency hospitalization have decreased; bed availability per capita has not increased and the number of beds available is just above 200 per day on average. Conclusions: hospitals with greater service capacity are the Leon Becerra, the Naval and the Military hospitals. The hospitals in the southern and western part of the city have smaller capacity to serve a larger number of patients.

KEYWORDS: emergency medical services, hospital administration, hospital bed capacity.

Resumo

Objetivos: descrever as características gerais que têm os serviços de emergência dos hospitais da cidade de Guayaquil; o fluxo de pacientes, as proporções de internamento e cirurgias de emergência, as taxas de ocupação e a disponibilidade de camas, estimando a possibilidade de atender a um súbito aumento de demanda por uma catástrofe natural, modelar uma situação de aumento pela estimativa da capacidade de atenção em função da disponibilidade de camas. Desenho do estudo: transversal para a descrição dos serviços de emergência de cinco hospitais na Província de Guayas e simular um aumento linear na procura de serviços para avaliar a capacidade de atenção. Resultados: os cuidados de emergência aumentaram significativamente ao longo dos últimos cinco anos, enquanto as taxas de internação de emergência diminuíram; a disponibilidade de leitos por habitante não aumentou e o número de leitos disponíveis é pouco mais que 200 por dia em média. Conclusões: os hospitais que têm a maior percentagem da capacidade de atenção são o Leon Becerra, o Naval e o Militar. Os hospitais no setor do sul e oeste da cidade são aqueles que têm uma menor capacidade para atender um volume maior de pacientes.

PALABRAS-CHAVE: serviços médicos de emergência, administração hospitalar, número de leitos em hospital. 
INTRODUCCIÓN

El Ecuador está en un territorio de riesgo sísmico, tanto por el movimiento de las placas tectónicas Continental y de Nazca, así como por una serie de volcanes activos. Existen registros de nueve sismos mayores a 6 grados de magnitud en un periodo de poco más de 200 años y cerca de 100,000 víctimas mortales. ${ }^{1}$ Para las atenciones médicas en la ciudad de Guayaquil se reconocen 85 establecimientos con hospitalización; y de estos, 38 reportaron disponer de área de emergencia.

El peligro de enfrentar un sismo grave ha llevado a organismos como la Organización Mundial de la Salud y la Organización de Naciones Unidas a planificar, en base a la experiencia, la ayuda en desastres naturales y la posible respuesta de los servicios médicos.

Frecuentemente se divide la posibilidad de daños en estructurales y no estructurales, y entre estos últimos los denominados daños funcionales, relacionados con los servicios de soporte, provisión y organización de recursos. ${ }^{2} \mathrm{Si}$ bien la complejidad de la relación de los recursos y servicios en un hospital, a más de la incertidumbre de arribo de pacientes, no permite alejarse de una mirada de conjunto. Es necesario precisar algún indicador que ayude a medir la capacidad de servicio, y el número de médicos es una de ellas. Este peligro sísmico conlleva a analizar los recursos disponibles y la operación de los servicios de emergencia.

El análisis de las operaciones hospitalarias ayuda a la comprensión no solamente de la disponibilidad y coordinación de recursos, sino además del flujo de pacientes, la interacción compleja de servicios y el impacto que tiene en los pacientes. ${ }^{3}$ Por lo que el objetivo de este estudio es determinar la capacidad de respuesta ante un incremento súbito de la demanda a los principales hospitales del Guayas; esto último utilizando herramientas de investigación, modelación y simulación de procesos de atención. ${ }^{4}$

\section{MÉTODOS}

Se hizo un tipo de estudio transversal para la descripción de los servicios de emergencia de los hospitales de la provincia del Guayas. Se revisó dos bases de datos del INEC, la de "actividades y recursos hospitalarios" y la correspondiente a "camas y egresos hospitalarios" de los años 2008 al 2012. Se tabuló los resúmenes y sumatorias de atención y se calculó el crecimiento de las atenciones, tanto en consultas de emergencia como en cirugías e internamientos del mismo tipo. Además se hizo una estimación de índices de intervenciones quirúrgicas e ingresos por cada 1,000 consultas. Los hospitales que se analizaron fueron León Becerra, Naval, Militar, Universitario y Luis Vernaza.

Se realizaron observaciones en cinco hospitales de tipo general, no especializados, durante 100 días desde el mes de abril del año 2013, con el objetivo de modelar el arribo de pacientes al AE, específicamente el número de arribos por hora, para luego estimar la distribución y los parámetros fundamentales.

De la base de datos de egresos hospitalarios del año 2012 se establecieron otras características de las condiciones normales de operación y sus volúmenes de atención, como son el porcentaje de ocupación promedio de cada uno de los cinco hospitales, el promedio de duración de la hospitalización, el promedio diario de camas disponibles y la proporción de pacientes que requieren internamiento, una vez que han sido atendidos en la emergencia. Estas características son susceptibles de modelación para ayudar en el análisis, mejora o diseño. ${ }^{5}$ Tales modelos pueden ser prescriptivos, descriptivos. ${ }^{6}$ Los primeros se pueden clasificar por los objetivos: de simulación o de optimización, orientados a mejorar el desempeño. ${ }^{7,8}$ Los modelos descriptivos ofrecen un reporte detallado de la conducta de la organización mediante representaciones tabulares, algebráicas o gráficas, entre las que se encuentran los modelos lineales o no lineales, siempre recogiendo las principales propiedades del sistema. ${ }^{9-11}$

Se revisaron los registros de eventos sísmicos en distintos países y los efectos en la demanda de servicios de emergencia y las características principales del flujo de pacientes. Para la simulación del comportamiento de las AE de los hospitales de Guayaquil se estimó el crecimiento de la cantidad de arribos en tres niveles, en base a experiencias previas con sismos de baja, mediana y alta intensidad, como reportaron Pengfei Yi y sus colaboradores, ${ }^{12}$ quienes bajo un modelo de regresión cuadrática estimaron el comportamiento del incremento de la demanda en tres posibles niveles. Los tres niveles que se escogieron arbitrariamente en este estudio fueron $10 \%, 30 \%$ y $70 \%$ para incrementar linealmente el número de arribos, al que adicionalmente se afectó con el porcentaje de internamiento por emergencia de cada hospital. 
RESULTADOS

El número de establecimientos de salud registrados formalmente en Ecuador en el año 2012 suma 4.015; de éstos, 735 cuentan con servicios de internación. En un período de cinco años, ambas cifras crecieron $4.3 \%$ y $0.8 \%$ respectivamente. La provincia del Guayas registra 549 establecimientos en total y 138 con internación, valores que representan $13.7 \%$ y $18.8 \%$ de los totales respectivos.

TABLA 1. ACTIVIDADES DE EMERGENCIA EN HOSPITALES DE LA PROVINCIA DEL GUAYAS, AÑOS 2008 AL 2012

\begin{tabular}{lrrrrr} 
& $\mathbf{2 0 0 8}$ & $\mathbf{2 0 0 9}$ & $\mathbf{2 0 1 0}$ & $\mathbf{2 0 1 1}$ & $\mathbf{2 0 1 2}$ \\
\hline $\begin{array}{l}\text { Número de } \\
\text { atenciones }\end{array}$ & 760.874 & 1414.416 & 1622.018 & 1595.060 & 2075.238 \\
\hline $\begin{array}{l}\text { Ingresos de } \\
\text { Emergencia }\end{array}$ & 245.063 & 198.787 & 129.282 & 132.616 & 156.670 \\
\hline $\begin{array}{l}\text { Cirugías de } \\
\text { Emergencia }\end{array}$ & 49.451 & 52.306 & 63.066 & 34.182 & 210.918 \\
\hline
\end{tabular}

En términos absolutos las atenciones crecieron $173 \%$, los ingresos decrecieron en $36 \%$, mientras que las cirugías se incrementaron en $327 \%$. En el año 2012 arribaron a los servicios de emergencia 3.95 pacientes por minuto en la provincia del Guayas, 2.5 de éstos solamente en Guayaquil. Los ingresos de emergencia en Guayaquil representan el $88 \%$ y las cirugías el $61 \%$ de todos los que se producen en la provincia (tabla 1). Con estas cifras se calculó la tasa de ingresos y cirugías entre cada mil atenciones de emergencia, siendo el año 2010 el de más baja tasa de ingresos y el año 2011 el más bajo de cirugías.

TABLA 2. TASA DE INTERNAMIENTO Y CIRUGÍAS POR CADA MIL ATENCIONES DE EMERGENCIA, PROVINCIA DEL GUAYAS

\begin{tabular}{lrrrrrr}
\hline TASA (T/1000) & $\mathbf{2 0 0 8}$ & $\mathbf{2 0 0 9}$ & $\mathbf{2 0 1 0}$ & $\mathbf{2 0 1 1}$ & $\mathbf{2 0 1 2}$ & $\begin{array}{r}\text { VARIACIÍN } \\
\mathbf{2 0 1 2} \text { vs. 2008 }\end{array}$ \\
\hline Internamientos & 322 & 141 & 80 & 83 & 75 & $-77 \%$ \\
\hline Cirugías & 65 & 37 & 39 & 21 & 102 & $56 \%$ \\
\hline
\end{tabular}

La tasa de internamiento por mil atenciones disminuye en $77 \%$, mientras que la de cirugías aumenta en $56 \%$ en la ciudad de Cuayaquil. Esta tasa es de 106.6, lo que significa que es $41 \%$ más alta que en la provincia, y la tasa de cirugías es 99, apenas menor que lo que sucede en otras ciudades. Esto para el año 2012 (tabla 2 y figura 1).

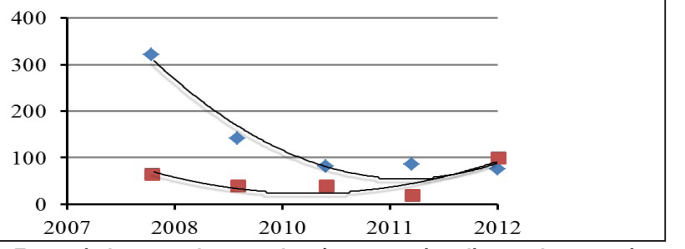

Figura 1. Tasas de internamiento y cirugías por cada mil atenciones en la provincia del Guayas.
Las observaciones realizadas en los cinco hospitales permitieron estimar los siguientes parámetros, que incluyen en la frecuencia acumulada (tabla 3 y figura 2).

\section{TABLA 3. MODELOS DE ARRIBO DE PACIENTES A LOS CINCO HOSPITALES EN AE}

\begin{tabular}{|c|c|c|c|c|c|c|c|c|}
\hline \multirow[b]{2}{*}{$\begin{array}{l}\text { 㢇 } \\
\text { 高 }\end{array}$} & \multicolumn{4}{|c|}{ ARRIBOS DIARIOS } & \multicolumn{4}{|c|}{ ARRIBOS POR HORA } \\
\hline & 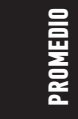 & 琶 & 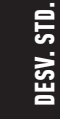 & بُ & 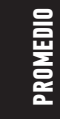 & 䇺蒠 & $\begin{array}{l}\text { 总 } \\
\text { 兽 }\end{array}$ & ب \\
\hline $\begin{array}{l}\text { Leon } \\
\text { Becerra }\end{array}$ & 38.63 & 0.59 & 5.86 & $\begin{array}{r}37.48- \\
39.78\end{array}$ & 1.61 & 0.13 & 1.26 & $\begin{array}{l}.36- \\
1.86\end{array}$ \\
\hline Naval & 25.50 & 0.60 & 5.95 & $\begin{array}{r}24.33- \\
26.67\end{array}$ & 1.01 & 0.10 & 1.03 & $\begin{array}{l}0.8- \\
1.21\end{array}$ \\
\hline Militar & 24.71 & 0.46 & 4.58 & $\begin{array}{r}23.81- \\
25.61\end{array}$ & 1.03 & 0.10 & 1.01 & $\begin{array}{r}0.83- \\
1.23\end{array}$ \\
\hline $\begin{array}{l}\text { Univer- } \\
\text { sitario }\end{array}$ & 71.80 & 0.93 & 9.26 & $\begin{array}{r}69.99- \\
73.62\end{array}$ & 3.12 & 0.17 & 1.67 & $\begin{array}{r}2.79- \\
3.45\end{array}$ \\
\hline $\begin{array}{l}\text { Luis } \\
\text { Vernaza }\end{array}$ & 75.06 & 0.88 & 8.75 & $\begin{array}{r}73.35- \\
76.78\end{array}$ & 3.12 & 0.18 & 1.76 & $\begin{array}{r}2.77- \\
3.46\end{array}$ \\
\hline
\end{tabular}

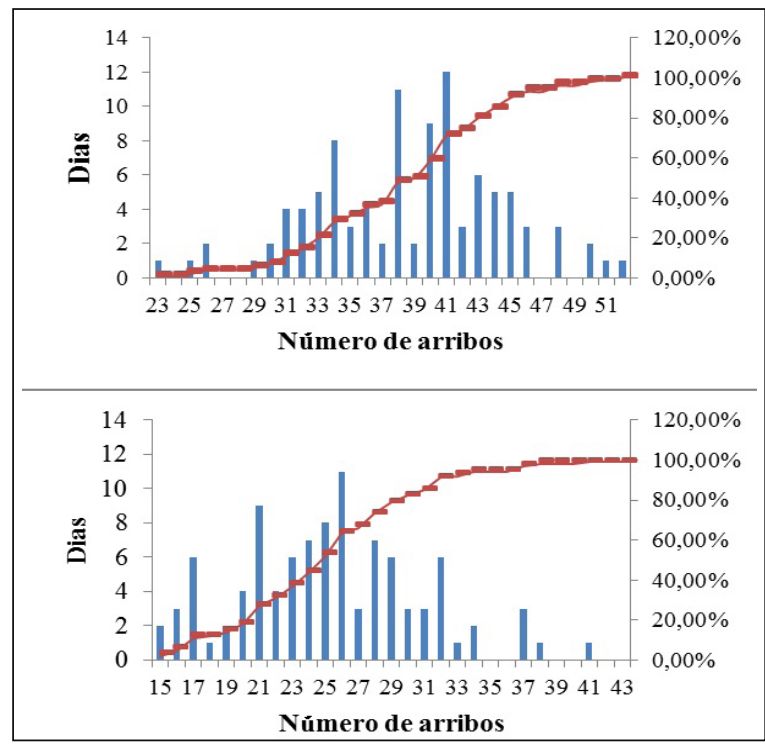

Figura 2. Histograma de arribos diarios en el área de emergencia de los hospitales León Becerra y Naval.

En 19 hospitales se encontró información completa que permite relacionar el número de determinaciones de laboratorio y el número de exámenes de imágenes por cada atención, resultando un índice de 4.45 y de 0.29 respectivamente. Hasta aquí, el estudio recoge información que ayuda a perfilar los volúmenes de atención de las $\mathrm{AE}$, a nivel general. No encontrándose información detallada para establecer más parámetros, como el tiempo de estadía de los pacientes ingresados por emergencia, la distribución de edades de los pacientes o una información acerca del tipo de especialidad que requieren los pacientes (figura 3). 


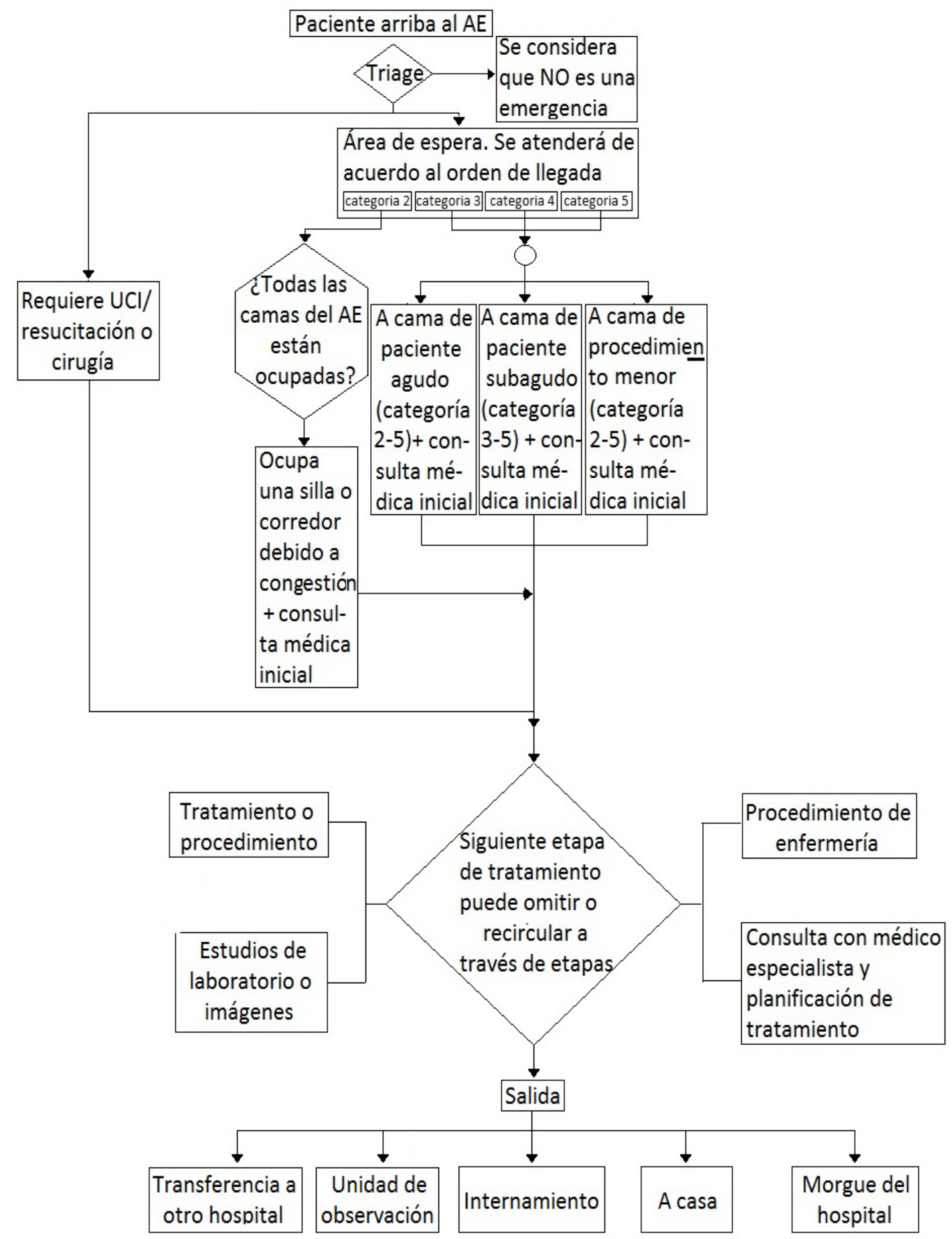

Figura 3. Modelo del flujo regular de pacientes en el AE. 
Se estimó la capacidad de atención hospitalaria en caso de un sismo grave y se compararon los parámetros de atención regular de las $\mathrm{AE}$, la tasa de ocupación promedio y el número de camas disponibles con las cifras incrementadas en tres rangos de arribo de pacientes: $10 \%, 30 \%$ y $70 \%$ (tabla 4).

\begin{tabular}{|c|c|c|c|c|c|c|c|}
\hline \multirow{3}{*}{ HOSPITAL } & \multicolumn{4}{|c|}{ CONDICIONES NORMALES } & \multirow{2}{*}{\multicolumn{3}{|c|}{$\begin{array}{l}\text { RANGO DE } \\
\text { INCREMENTO } \\
\text { ESTIMADO PARA } \\
\text { INTERNAMIENTO (\# } \\
\text { DE PACIENTES) }\end{array}$}} \\
\hline & \multirow{2}{*}{ 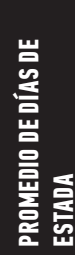 } & \multirow{2}{*}{ 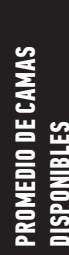 } & \multirow{2}{*}{ 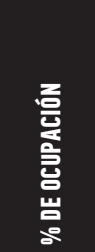 } & \multirow{2}{*}{ 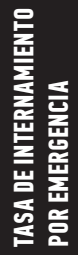 } & & & \\
\hline & & & & & $\stackrel{\circ}{\circ}$ & 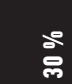 & $\stackrel{\circ}{\circ}$ \\
\hline $\begin{array}{l}\text { León } \\
\text { Becerra }\end{array}$ & 5 & 57 & $42.49 \%$ & 0.13 & 0.50 & 1.51 & 3.52 \\
\hline Naval & 4.4 & $42-2 \cdot 3 \cdot 2 \cdot 2 \cdot$ & $41.99 \%$ & 0.04 & 0.10 & 0.29 & 0.68 \\
\hline Militar & 3.9 & 17 & $34.46 \%$ & 0.03 & 0.07 & 0.22 & 0.52 \\
\hline $\begin{array}{l}\text { Universi- } \\
\text { tario }\end{array}$ & 4.2 & 19 & $85.23 \%$ & 0.32 & 2.40 & 7.19 & 16.77 \\
\hline $\begin{array}{l}\text { Luis } \\
\text { Vernaza }\end{array}$ & 8.2 & 82 & $87.27 \%$ & 0.66 & 4.94 & 14.82 & 34.58 \\
\hline
\end{tabular}

Los hospitales que tendrían mayor capacidad de porcentaje de camas disponibles y número de días con capacidad de internamiento son el Naval, el Militar y el León Becerra. El hospital Luis Vernaza es el hospital con mayor número de camas disponibles, sin embargo presenta porcentualmente la menor tasa de desocupación de camas (tabla 4 y 5).

\begin{tabular}{lccr}
\hline TABLA 5. NÚMERO DE DÍAS CON CAPACIDAD DE INTERNAMIENTO \\
PARA DISTINTOS RANGOS DE CRECIMIENTO DE DEMANDA \\
\hline HOSPITAL & $\mathbf{1 0 \%}$ & $\mathbf{3 0 \%}$ & $\mathbf{7 0 \%}$ \\
\hline León Becerra & 114 & 38 & 16 \\
\hline Naval & 436 & 145 & 62 \\
\hline Militar & 229 & 76 & 33 \\
\hline Universitario & 8 & 3 & 1 \\
\hline Luis Vernaza & 17 & 6 & 2 \\
\hline
\end{tabular}

DISCUSIÓN

La capacidad de atención de las Áreas de Emergencia (AE) de los hospitales en el Ecuador está sometida a presión permanentemente. En el año 2012 se brindaron 9 millones de atenciones de emergencia, equivalente a 581 por cada mil habitantes, tasa que representa el triple de la registrada en el año 2008. ${ }^{13}$ Cada minuto se atendió a 17 pacientes en los servicios de emergencia en Ecuador, 4 de ellos en la provincia del Guayas. Las camas hospitalarias por cada mil habitantes muestran en cambio una tasa de 1.6 en el año 2008 y de 1.5 en el $2012 .{ }^{14} \mathrm{La}$ congestión hospitalaria es uno de los resultados del incremento de las tasas de atención y en las $\mathrm{AE}$ lo que provoca largos tiempos de espera. ${ }^{15}$

En el análisis presente se determinó que las tasas de arribo a las AE son asimétricas entre los cinco hospitales estudiados. El hospital Vernaza tiene el promedio de estadía de pacientes internados más largo y el León Becerra el más corto. Los porcentajes de ocupación mayor y menor lo tendrían el Luis Vernaza y Naval respectivamente. Los resultados del modelo de incremento de pacientes que requieran una atención de emergencia, luego de un sismo de mayor intensidad, sugieren que probablemente los hospitales León Becerra, Naval y Militar tengan mayor capacidad de atención. Los hospitales del sector del sur y oeste de la ciudad son los que tendrían menor capacidad de incremento para atender un mayor volumen de pacientes.

Frente a un sismo de mayor intensidad la evaluación de la mano de obra de los médicos ha sido llevada a cabo para bosquejar una mejor respuesta en la recepción de pacientes, como lo mencionan Chen y colaboradores. ${ }^{16}$

En este estudio se revisaron los expedientes de los pacientes, la carga de trabajo de los médicos antes y después de un terremoto de gran magnitud. La movilización de los médicos en la respuesta de emergencia posterremoto se evaluó mediante un cuestionario confidencial y se encontró que en los tres días posteriores al terremoto, 566 pacientes con enfermedades o lesiones relacionadas con el terremoto fueron enviados a la emergencia del hospital.

El tipo de servicio y el incremento en su utilización después de un sismo, incluyendo el tiempo promedio de atención en la emergencia hospitalaria, lo investigó Shimada y sus colaboradores, ${ }^{17}$ encontrando mayor número de pacientes, menor tiempo promedio en la atención, incremento de tasa de transfusiones y mayor demanda de medicamentos.

El esquema de atención se puede resumir en una etapa de arribo al área de triaje, en donde 
se valora al paciente y se orienta el posterior examen médico. Éste generalmente requiere la realización de exámenes de laboratorio, de imágenes o el inicio de un tratamiento farmacológico. Existe un periodo de espera hasta que se completen los resultados de los exámenes y luego se toma la decisión de brindar un cuidado adicional. En caso de no ameritarlo, el paciente es dado de alta y en caso de requerirlo, se plantean las alternativas de admisión o de traslado a otra unidad de salud (figura 3).

Una limitación del estudio ha sido que la disponibilidad de información del tipo de consulta de emergencia atendida es limitada o no está registrada o publicada. Otra limitación es que se ha puesto a un lado factores como la disponibilidad de otros recursos, como podrían ser el número de horas médico disponible para las AE o la disponibilidad de recursos materiales e insumos necesarios para atender a los pacientes.

\section{CONCLUSIONES}

Algunos de los servicios de emergencia en los hospitales de la ciudad de Guayaquil podrían comenzar a presentar saturación en la capacidad de internamiento al segundo día si se observa un incremento en la demanda en un $70 \%$. Para ello se requiere información que permita estudiar las especialidades más frecuentemente demandadas por los pacientes, tiempos de espera, los tiempos quirúrgicos, todo esto con la finalidad de eficientizar más los servicios de cada hospital y ayudar a proponer alternativas de gestión de procesos que reduzcan la congestión, aumenten la satisfacción de los usuarios y permitan estar preparados para enfrentar un aumento de la demanda por un desastre natural $u$ otro tipo de emergencia.

\section{REFERENCIAS BIBLIOGRÁFICAS}

3. Tekwani K, KY, MC, SB, \& KE. Emergency Department Crowding is Associated with Reduced Satisfaction Scores in Patients Discharged from the Emergency Department. Western Journal of Emergency Medicine. 2012; 11(15).

4. De Lia D, \& CJ. Emergency department utilization and capacity. Robert Wood Johnson Foundation. 2009.

5. Robinson S. Tutorial: Choosing what to modelconceptual modeling for simulation. Winter Simulation Conference. 2012.

6. Sinreich D, Marmor Y. Emergency department operations: The basis for developing a simulation tool. IIE Transactions. 2005; 37.

7. Ades AE, \& CS. Markov Chain Monte Carlo Estimation of a Multiparameter Decision Model: Consistency of Evidence and the Accurate Assessment of Uncertainty. Medical Decision Making. 2002; 22: p. 359 - 371.

8. Sharoda P, \& MR. A Systematic Review of Simulation Studies Investigating Emergency Department Overcrowding. Simulation. 2010.

9. Barros O, Wr, RC,FE,\&JC. Demand Forecasting and Capacity Planning for Hospitals. Departamento de Ingeniería Industrial, Universidad de Chile. 2012.

10. Lakshmi C, \& SA. Application of queueing theory in health care: a literature review. Operations research for health care. 2013.

11. Saghafian S, AG, \& TS. Social Science Research Network. [Online].; 2014 [cited 2014 enero 3. Available from: http://www.ssrn.com/en/.

12. Yi P, Santhosh G, Paul J, Lin L. Hospital capacity planning for disaster emergency management. Socio-Economic Planning Sciences. 2010 septiembre; 44 (3).

13. INEC. Anuario de recursos y actividades de salud INEC, editor. Quito: INEC; 2012.

14. INEC. Anuario de camas y egresos hospitalarios Quito: INEC; 2012

15. Bernstein S, VV, LW, LA, \& PI. Development and Validation of a New Index to Measure Emergency Department Crowding. Acad Emerg Med. 2003; 10 (9).

16. Chen Wk, Chen YC, Choy K, Hung JCM. Were There Enough Physicians in an Emergency Department in the Affected Area After a Major Earthquake? An Analysis of the Taiwan Chi-Chi Earthquake in 1999. Annals of Emergency Medicine. 2001 julio; 38(5).

17. Shimada M, Gunshin M, Tanabe A, Riffenburgh RH, Tanen DA. Resource Utilization in the Emergency Department of a Tertiary Care University-Based Hospital in Tokyo Before and After the Sendai Earthquake and Tsunami. Annals of Emergency Medicine. 2011; 58(4). 